\title{
Bilan et perspectives de la gestion de la variabilité génétique des ovins laitiers en France
}

INRA Prod. Anim.

\author{
Diane BUISSON' ${ }^{1}$, Jean-Michel ASTRUC' ${ }^{1}$, Francis BARILLET ${ }^{2}$ \\ ${ }^{1}$ Institut de l'élevage, CNBL, 31321, Castanet-Tolosan, France \\ ${ }^{2}$ GenPhySE, Université de Toulouse, INRA, INPT, INP-ENVT, 31320, Castanet Tolosan, France \\ Courriel:diane.buisson@toulouse.inra.fr
}

Le déploiement de la sélection génomique en Ovins Laitiers en France pose la question des risques d'accroissement de la consanguinité dans un schéma où le turn-over des mâles élites est plus rapide. Un bilan de la gestion de la variabilité génétique a donc été dressé dans les cinq races ovines laitières françaises pour faire le point sur l'état de ces populations et, si besoin, travailler avec les entreprises de sélection sur la gestion des mâles élites et des accouplements raisonnés.

\section{Introduction}

En bovin lait, en raison de l'essor de quelques races à l'échelle mondiale (Prim'Holstein et Jersiaise notamment) et du développement du «star system », qui s'appuie sur un usage très déséquilibré d'un nombre limité de taureaux stars, le niveau de consanguinité a fortement augmenté au cours des dernières décennies, provoquant une forte perte de diversité génétique (Sørensen et al., 2005) et l'apparition de tares génétiques. La consanguinité moyenne atteint ainsi $6 \%$ dans les populations canadiennes Holstein et Jersiaises en 2008 (Stachowicz et al., 2011).

À l'inverse, en France, les éleveurs d'Ovins Laitiers (OL) ont fait le choix de sélectionner des races pures, locales, dans leurs bassins respectifs de production. L'amélioration génétique s'appuie sur des schémas de sélection présentant un gain génétique annuel important compris entre 0,10 et 0,23 écart-type génétique (Astruc et al., 2016). L'efficacité de ces schémas tient à leur organisation pyramidale, qui permet la sélection des mâles par testage sur descendance chez les éleveurs du noyau de sélection en Contrôle Laitier Officiel (CLO), et à l'utilisation de I'Insémination Artificielle (IA) pour créer et diffuser largement le progrès génétique. La pratique de I'IA en semence fraîche et la forte saisonnalité de la campagne d'IA impliquent de disposer d'un pool de béliers vivants important, suffisant pour répondre au besoin, ce qui peut permettre une utilisation aussi équilibrée que possible des béliers élites, avec toutefois une capacité de diffusion plus ou moins limitée selon les races : jusqu'à 1200 IA par bélier et par an en Lacaune Lait (LL), vs 120 à 500 IA par bélier et par an dans les Races Ovines Laitières des Pyrénées (ROLP) ou la race Corse (C). Cependant, si des règles de gestion de la variabilité génétique ne sont pas mises en œuvre, cette dernière va s'éroder sous les effets de ces schémas de sélection efficaces.

Afin de mesurer l'impact de la gestion de la variabilité génétique mise en œuvre dans ces races, deux études rétrospectives avaient été menées à 10 ans d'intervalle : la première par
Barillet et al. (1989) et la seconde par Palhière et al. (2000). Or, suite à l'épisode de tremblante ayant touché les races ovines françaises dans la décennie 1990, les gestionnaires des schémas ont été amenés à sélectionner plus ou moins rapidement, dans la décennie 2000 , des reproducteurs homozygotes résistants à la tremblante pour le gène majeur PrP, en particulier les béliers des centres d'insémination animale (Barillet et al., 2004). Il est donc légitime de s'interroger sur l'apparition éventuelle de goulots d'étranglements liés à la sélection sur le gène PrP (Brochard et al., 2006) et des conséquences négatives possibles sur l'évolution de la variabilité génétique.

Par ailleurs, avec l'arrivée de la Sélection Génomique (SG), les Entreprises de Sélection (ES) peuvent diminuer la taille du haras de béliers $d^{\prime} I A$, avec la suppression de la catégorie des béliers d'IA en attente des résultats de testage (schéma classique), tout en cherchant simultanément à maximiser le nombre d'IA par mâle, dans le but de compenser les coûts supplémentaires engendrés par le génotypage d'un 
grand nombre d'agneaux et leur entretien en centre d'élevage. De plus, I'utilisation de béliers élites génomiques devrait réduire l'intervalle de générations sur la voie père-fils et permettre de dégager environ $15 \%$ de progrès génétique supplémentaire (Buisson et al., 2014). C'est donc dans ce contexte que se pose désormais la question de l'efficacité de la gestion de la variabilité génétique des noyaux de sélection des races ovines laitières françaises.

L'objectif de la présente étude consiste à dresser un bilan de la gestion de la variabilité génétique pour chacune des cinq races ovines laitières françaises au cours des 40 dernières années, afin d'en apprécier l'efficacité et, si besoin, de faire évoluer les règles de gestion mises en œuvre.

\section{1. Éléments démographiques et organisationnels des schémas de sélection}

\section{1. Évolution et pérennité des bases de sélection au cours des quatre dernières décennies}

Les schémas de sélection ovins laitiers français classiques et maintenant génomiques ont été décrits précisé-

Tableau 1. Caractéristiques générales des différents schémas de sélection ovins laitiers en France en 2014 (Idele et CNBL, 2015).

\begin{tabular}{|c|c|c|c|c|c|c|}
\hline & $\begin{array}{c}\text { Taille de la } \\
\text { population } \\
\text { o }\end{array}$ & $\begin{array}{c}\text { Noyau } \\
\text { de selection } \\
\text { ( } \mathbf{( \% )}\end{array}$ & $\begin{array}{c}\boldsymbol{\lambda} \text { en } \\
\text { testage }\end{array}$ & $\begin{array}{c}\text { o en } \\
\text { centre } \\
\text { d'IA }\end{array}$ & $\begin{array}{c}\text { PAB en } \\
\text { centre } \\
\text { d'IA }\end{array}$ & $\begin{array}{c}\text { IA dans } \\
\text { le noyau } \\
\text { (\%) }\end{array}$ \\
\hline LL & 890000 & 19 & 440 & 1400 & $70-80$ & 85 \\
\hline MTR & 274000 & 29 & 150 & 600 & 30 & 60 \\
\hline BB & 80000 & 32 & 50 & 200 & 17 & 55 \\
\hline MTN & 80000 & 16 & 30 & 175 & 17 & 45 \\
\hline CORSE & 83000 & 19 & 30 & 150 & 22 & 40 \\
\hline
\end{tabular}

ment par Astruc et al. (2016). Les schémas classiques sont organisés selon une structure pyramidale de la population s'appuyant chaque année sur le testage sur descendance d'un grand nombre de béliers d'IA (Astruc et al., 2010) : 150 à 250 en Manech Tête Rousse (MTR) et dans chacune des 2 ES Lacaune, et 25 à 40 dans les races Corse, Manech Tête Noire (MTN) et Basco-Béarnaises (BB). Actuellement, le noyau de sélection représente 20 à $30 \%$ des brebis de chacune des races (tableau 1), mais les effectifs de sélectionneurs et de brebis ont fortement évolué au cours des dernières décennies.

Si on s'intéresse tout d'abord à la race Lacaune, il apparaît un doublement du nombre d'éleveurs sélectionneurs entre 1972 et 1975 avec l'arrivée massive de nouveaux élevages (figure 1a). Cet accroissement rapide découle de la scission du noyau de sélection de la Confédération Générale de Roquefort, dont un tiers environ est parti pour la création d'une seconde ES en 1972, la coopérative Ovitest. Après une période de stabilisation, le nombre de sélectionneurs augmente à nouveau entre 1983 et 2004 pour passer de 310 à 400 environ, puis diminue légèrement chaque année depuis 2005 pour atteindre 366 en 2016 répartis en 2 ES de même taille, le Service Élevage de la Confédération Générale de Roquefort et la coopérative Ovitest. Des mouvements très limités d'éleveurs sélection-

Figure 1. Évolution du nombre d'éleveurs sélectionneurs (Contrôle Laitier Officiel CLO), de 1970 à 2012, dans les trois bassins laitiers français (1a), et pour chacune des trois races ovines laitières des Pyrénées (1b).

$1 \mathrm{a}$

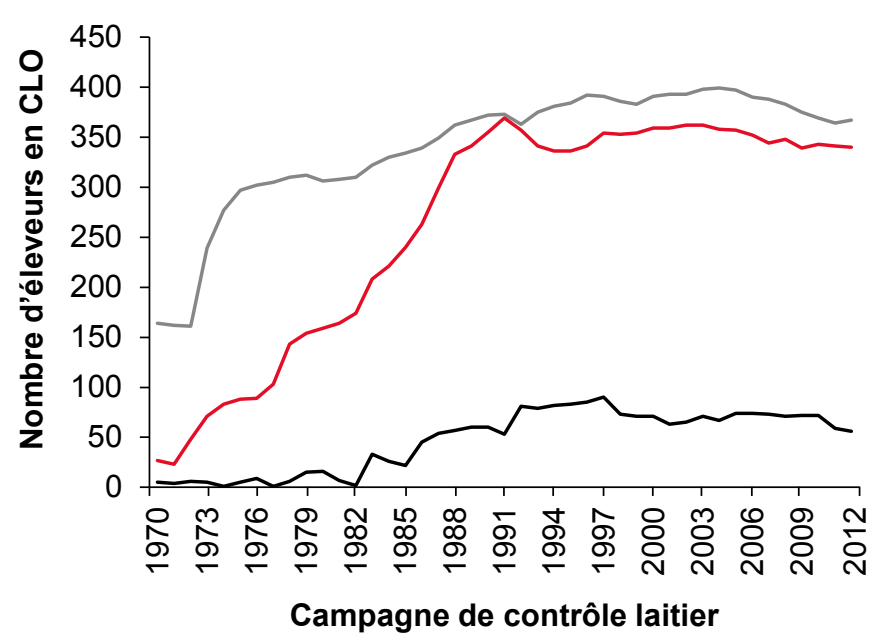

_ Rayon de Roquefort

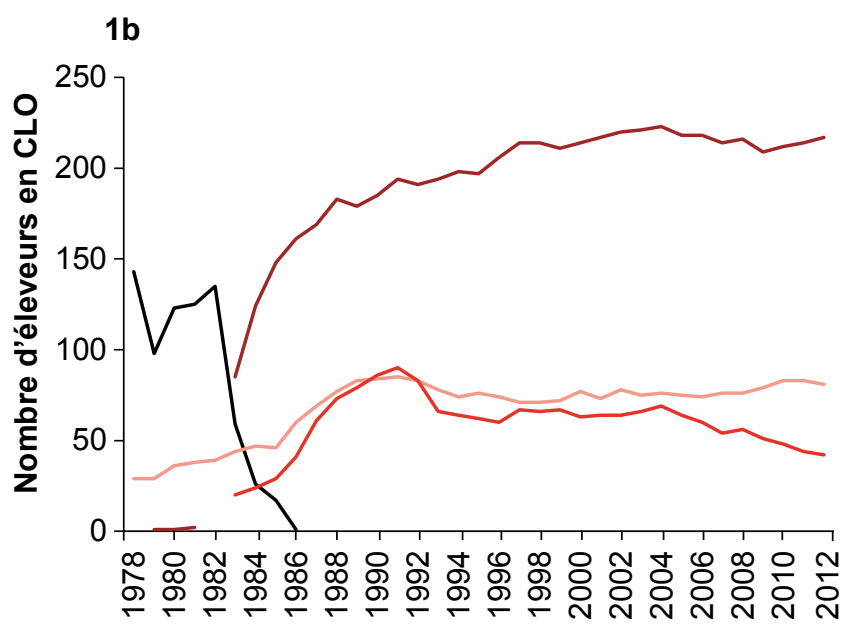

Campagne de contrôle laitier

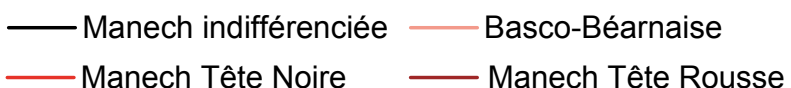


neurs, entrées et sorties, sont observés chaque année, avec des échanges entre les deux ES qui restent marginaux.

Dans les Pyrénées, le nombre d'éleveurs sélectionneurs augmente fortement jusqu'au début des années 1990, suite au démarrage du schéma de sélection dans les années 1980. Les effectifs se stabilisent ensuite autour de 350 sélectionneurs jusqu'au début des années 2000 et, comme en LL, tendent à baisser légèrement depuis pour atteindre 336 en 2016. Les mouvements d'entrée/sortie d'élevages sélectionneurs sont plus importants dans ce bassin que dans le Rayon de Roquefort (figure 1a). L'analyse par race montre que le nombre de sélectionneurs est relativement stable en races BB et MTR depuis 1990 (environ 75 et 215 respectivement), en revanche, leur nombre se réduit en race MTN avec une première baisse ponctuelle au début des années 90 , liée à la refonte des contrats de sélection (on passe de 90 à 65 sélectionneurs), et une baisse continue depuis 2004 pour atteindre 35 sélectionneurs en 2016 (figure 1b). En effet, la MTN est en recul et de nombreux éleveurs choisissent de changer de race et de passer à la BB ou la MTR pour des raisons économiques.

Enfin, en race Corse, le nombre de sélectionneurs augmente jusqu'à un effectif de 1990, du début des années 1980 jusqu'au milieu des années 1990, ce qui correspond au démarrage du schéma de sélection (figure 1a). En 1998 , suite à la refonte des contrats des sélectionneurs, une quinzaine d'éleveurs quitte le CLO. Les effectifs se stabilisent ensuite puis baissent légèrement à partir de 2010 pour atteindre 57 sélectionneurs en 2016.

Quelle que soit la race étudiée, les évolutions des effectifs de brebis par millésime (année de naissance) suivent les évolutions du nombre de sélectionneurs (figure 2). Durant la phase de démarrage du schéma de sélection, les effectifs s'accroissent fortement, avec une hausse plus marquée en LL du fait du dédoublement du noyau de sélection en 1972. Les effectifs continuent ensuite de s'accroître beaucoup plus lentement avec l'augmentation progressive de la taille
Figure 2. Évolution du nombre de brebis en sélection (CLO), par millésime (année de naissance), pour chacune des cinq races ovines laitières françaises.

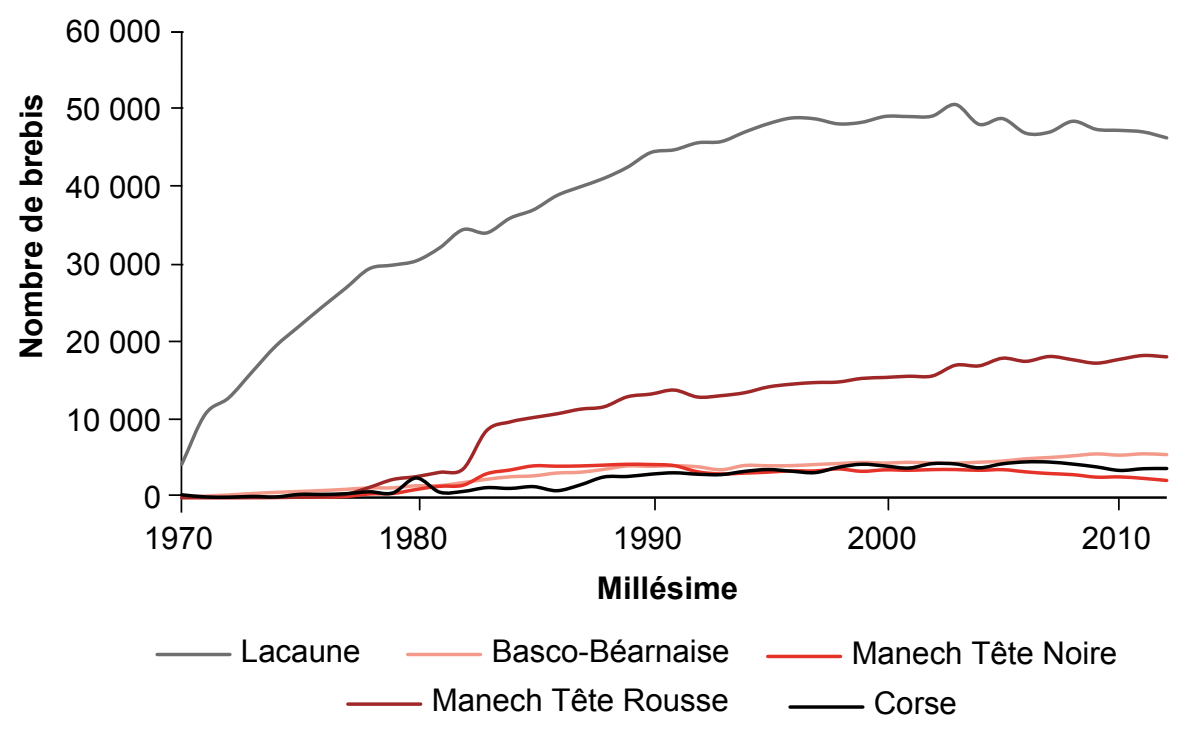

des troupeaux. Ces dernières années, les effectifs en CLO se stabilisent car, bien que la taille des troupeaux continue d'augmenter, le nombre de sélectionneurs diminue légèrement. Pour le millésime 2016, on compte environ 48020 brebis LL, 5210 brebis BB, 17200 brebis MTR, 1570 brebis MTN et 3590 brebis Corse.

\subsection{Les étapes clés de sélection des béliers du noyau de sélection}

Tous les ans, les jeunes béliers issus d'accouplements raisonnés (Pères à Béliers x Mères à Béliers) des élevages du noyau de sélection entrent en Centre d'Élevage (CE) à l'âge de 1 à 2 mois. Vers l'âge de 8 ou 18 mois selon les races, à l'issue d'un choix sur des critères fonctionnels, de standard et développement, et de fonction sexuelle, ces béliers sont mis en testage (schéma classique) ou participent à l'entretien de la population de référence (schéma génomique). Seuls les meilleurs béliers connus sur index sur descendance (ou sur index génomique pour une fraction d'entre eux) peuvent ensuite être utilisés comme béliers élites, c'est-à-dire comme Pères à Béliers $(\mathrm{PAB})$, pour procréer les futurs béliers d'IA, grâce aux accouplements raisonnés avec les meilleures femelles du noyau de sélection (Mères à Béliers). Le tableau 1 présente les principaux chiffres des schémas de sélection OL.
La sélection des OL s'appuie donc sur plusieurs étapes de sélection importantes : le tri des agneaux mâles qui entrent en $C E$, le choix des jeunes béliers à mettre en testage dans le schéma classique (30 à 440 selon la race) ou à I'IA pour l'entretien de la population de référence dans le schéma génomique (Lacaune depuis 2015, ROLP depuis 2017), le choix des PAB (17 à 80 selon la race) et des femelles élites, et enfin l'organisation des accouplements raisonnés.

\subsection{Historique de la gestion de la variabilité génétique en ovins laitiers}

En 1965, les 100 meilleures brebis Lacaune avaient été accouplées avec les deux béliers les mieux indexés de l'année précédente (premiers calculs d'index réalisés en 1964) pour amorcer le schéma de sélection avec des béliers aussi améliorateurs que possibles. La diffusion massive de ces deux béliers a généré un goulot d'étranglement qui aurait pu provoquer un appauvrissement important de la variabilité génétique, si des règles de gestion strictes de celle-ci n'avaient pas été mises rapidement en place par les gestionnaires du schéma de sélection (Barillet et al., 1989).

À cet effet, la notion de familles, regroupant les descendants d'un même ancêtre, a donc été développée pour constituer des groupes de reproduction 
composés initialement d'élevages appartenant à une même zone géographique. L'arrivée de I'IA et le regroupement des béliers reproducteurs dans des centres $\mathrm{d}^{\prime} \mathrm{IA}$ a rendu impossible cette gestion géographique : les deux ES Lacaune ont donc pris le parti de gérer les mâles d'IA par familles (de Rochambeau et al., 2003). II s'agit donc d'appliquer une sélection intra-famille, l'idéal étant que chaque famille soit représentée par un seul PAB utilisé en IA et que celui-ci soit remplacé comme $P A B$ par un fils $d^{\prime} I A$ et un seul. La première ES organise des familles de Grands-Pères Paternels (GPP), soit 25 à 30 familles constituées $d^{\prime} 1$ à 2 fils par GPP (notion de titulaire et éventuellement d'un suppléant à cause de la pratique de I'IA en semence fraîche), familles qui sont reconstituées de façon dynamique chaque année et évoluent donc au fil du temps au gré des apparitions et disparitions de nouvelles familles élites. La seconde ES, quant à elle, établit des familles à partir de souches dites « éclatées » par rapport aux souches initiales, regroupant les descendants mâles d'un même arrière-GPP. Pour chacune des deux ES Lacaune, il y a donc chaque année 20 à 30 familles d'IA de béliers élites, comprenant chacune 1 à $2 \mathrm{PAB}$, soit un total par année d'environ 30 à 40 PAB utilisés en IA par chacune des deux ES.

Parallèlement à cette gestion par familles, de nouvelles règles ont été adoptées dans les années 1975. Tout d'abord, les accouplements entre individus partageant un ancêtre commun sur deux générations, c'est-à-dire parmi les parents et grands-parents, sont évités : on parle d'accouplements « interdits ». Toutefois, la règle essentielle, opérationnelle en IA en semence fraîche, correspond bien au fait que les PAB doivent être utilisés de manière équilibrée à chaque étape de la sélection : nombre d'accouplements raisonnés équilibrés entre les mâles élites, nombre équilibré d'agneaux sélectionnés par PAB pour l'entrée en centre d'élevage, nombre équilibré d'agneaux mis en testage par $P A B$. Ces règles de gestion ont ensuite été adoptées par les ROLP et la race Corse. Cependant, la plus petite taille des noyaux de sélection $B B$, MTN et $C$, et surtout la durée plus courte de la campagne $d^{\prime} \mid A$ dans ces trois dernières races rendent plus difficile le respect de la constitution de familles élites constituées uniquement de 1 ou 2 PAB. En effet, la durée trois fois plus courte de la campagne d'IA des ROLP comparativement à la race Lacaune tend à favoriser l'usage des PAB aptes à I'IA en semence fraîche dès le début de la campagne. Au fil du temps, I'apparition de déséquilibres plus ou moins marqués entre familles de PAB risque donc de s'installer. C'est effectivement ce qui s'est passé dans les années 2000 : si le nombre de PAB utilisés en moyenne chaque année est en rapport avec la taille de chacun des noyaux de sélection des races $B B, M T N, M T R$ et $C$, respectivement $17,17,30$ et $22 \mathrm{PAB}$ pour la campagne d'IA (tableau 1), en revanche ces $P A B$ ne représentent que 4 à 5 familles en BB, 5 à 6 familles en MTN, une douzaine en MTR et une dizaine en C. Il existe donc des familles élites d'IA constituées de nettement plus de deux $P A B$, particulièrement en races $B B$ et MTN.

\section{Les données analysées}

\subsection{Les populations analysées}

La présente étude se base sur l'analyse de données généalogiques des brebis et béliers du noyau de sélection, c'est- à-dire de reproducteurs des seuls élevages en CLO. Deux bases de données ont été constituées : une de « béliers » constitué des mâles d'IA et de monte naturelle ayant procréé au moins cinq filles en CLO, chacune d'elles ayant au moins une lactation non nulle, et une base « $P A B$ » constituée des béliers élites ayant au moins un fils dans la première base «béliers". L'analyse généalogique présentée concerne donc les béliers reproducteurs de chaque noyau de sélection.

En CLO, la majorité du renouvellement des femelles est issu de béliers d'IA en LL et ROLP (93\% en LL et $69 \%$ en ROLP, Idele et CNBL, 2017), et il représente la moitié en Corse (48\%, Idele et CNBL, 2017). De plus, bien que les accouplements en monte naturelle ne soient en général pas contrôlés en $\mathrm{CLO}$, on sait que les béliers de monte naturelle utilisés en CLO sont quasi tous nés de pères $d^{\prime} \mid A$ et de mères à béliers toutes issues d'IA. Ainsi, l'évolution de la variabilité génétique des brebis de chacun des noyaux de sélection dépend strictement de l'évolution de la variabilité génétique des béliers $d^{\prime} \mid A$, pour les 5 races de brebis laitières étudiées.

Les principaux résultats présentés ici sont donc ceux issus de l'analyse des

Tableau 2. Description des populations, issues du noyau de sélection (Contrôle Laitier Officiel - CLO, 1957-2013), de brebis ayant au moins une lactation non nulle ${ }^{1}$, de béliers ayant au moins 5 filles en CLO avec une lactation non nulle $(I A+M N)^{2}$, et de $P A B$ avec au moins 1 fils ayant au moins 5 filles en CLO avec une lactation non nulle $(I A+M N)^{3}$, et ayant été utilisées pour la création des bases de données étudiées.

Les effectifs totaux sont suivis des effectifs moyens par année de naissance dans le schéma en régime de croisière.

\begin{tabular}{|c|c|c|c|c|}
\hline & $\begin{array}{c}\text { Nombre } \\
\text { de brebis }\end{array}$ & $\begin{array}{c}\text { Nombre } \\
\text { de béliers }\end{array}$ & $\begin{array}{l}\text { Nombre } \\
\text { de } \text { PAB }^{(3)}\end{array}$ & $\begin{array}{c}\text { Régime } \\
\text { de croisière }\end{array}$ \\
\hline LL & $1617169(40000)$ & 19896 (492) & $1755(55)$ & \multirow{3}{*}{$\begin{array}{c}\text { Fin des années } \\
1980\end{array}$} \\
\hline LL_ES1 & $840449(20000)$ & $10269(225)$ & $925(25)$ & \\
\hline LL_ES2 & $776720(20000)$ & $9623(267)$ & $830(30)$ & \\
\hline BB & $130572(4500)$ & $1811(63)$ & $194(10)$ & \multirow{3}{*}{$\begin{array}{c}\text { Fin des années } \\
1990\end{array}$} \\
\hline MTN & 148506 (2 800) & $1031(35)$ & 126 & \\
\hline MTR & 458753 (15 400) & $6734(228)$ & $525(30)$ & \\
\hline C & $136296(3600)$ & $539(28)$ & $124(10)$ & $\begin{array}{c}\text { Début } \\
\text { des années } 2000\end{array}$ \\
\hline
\end{tabular}


données généalogiques des béliers reproducteurs (mâles d'IA et de monte naturelle) de chaque noyau de sélection, et du sous-ensemble des béliers des PAB d'IA. En outre, ce choix est également motivé par le fait que le niveau moyen de consanguinité et son évolution est comparable pour les brebis et les béliers reproducteurs du noyau de sélection, dès que la qualité de l'information généalogique est comparable dans les deux sexes, ce qui est le cas en race $L L$ à partir des années 90 (Palhière et al., 2000), et en ROLP à partir des années 2000. Les effectifs analysés sont présentés dans le tableau 2.

\subsection{Connaissance des généalogies}

La présente analyse de la variabilité génétique repose sur l'analyse des données généalogiques. Les résultats et interprétations sont donc d'autant plus fiables que l'on a du recul sur les généalogies, i.e. que les pedigrees sont profonds : plus on est capable de remonter dans les généalogies, plus il est possible de tenir compte d'ancêtres communs entre individus, et mieux la réalité peut être décrite, et donc plus la consanguinité et la parenté augmentent. Il est donc important de tenir compte de la connaissance des généalogies pour comparer les résultats entre races. Pour déterminer la profondeur des pedigrees, il est possible de calculer le nombre d'équivalent de générations connues (Ngen) qui est la somme des coefficients $(1 / 2)^{n}$ sur tous les ancêtres connus, n'étant le rang d'ascendance de l'ancêtre. Cet indicateur Ngen s'interprète ainsi : la qualité des généalogies de la population analysée est équivalente à celle d'une population pour laquelle, sur un nombre de génération égale à Ngen, tous les ancêtres seraient connus.

La connaissance des généalogies dépend donc de l'ancienneté des schémas de sélection et de la qualité de la collecte des généalogies : plus le recul sur la sélection de la race considérée est important et plus Ngen augmente (figure 3). La race $L L$, dont les deux schémas de sélection atteignent leur régime de croisière dans les années 1980, présente les pedigrees les plus profonds avec environ 10 équivalentgénérations connues pour les animaux nés en 2012 , ou encore $50 \%$ des béliers nés entre 2000 et 2009 sont connus sur au moins 9 générations (figure 4). De même, les ROLP sont relativement bien connues, avec 8 équivalent-générations connues pour les béliers MTR et BB nés en 2012 versus 6 pour les béliers MTN, et $50 \%$ des béliers nés entre 2000 et 2009 connus sur 5 à 7 générations. A contrario, les pedigrees Corse sont

peu profonds en raison du démarrage plus tardif du schéma de sélection (régime de croisière atteint dans les années 2000), mais également aussi à cause des mouvements d'éleveurs (entrées/sorties) du CLO qui entraînent des pertes d'information. Moins de 4 équivalent-générations sont connus en 2012 , et $50 \%$ des béliers nés entre 2000 et 2009 sont connus sur 3 générations (i.e. uniquement jusqu'aux arrièregrands-parents) en race Corse.

Figure 3. Évolution de la connaissance des pedigrees des béliers du noyau de sélection, nés de 1970 à 2013 par le calcul du nombre d'équivalents de générations connues (Ngen), dans les races ovines laitières Lacaune (LL), Basco-Béarnaise (BB), Manech Tête Noire (MTN), Manech Tête Rousse (MTR), Corse (C), et dans chacune des deux entreprises de sélection Lacaune (LL_ES1 et LL_ES2).

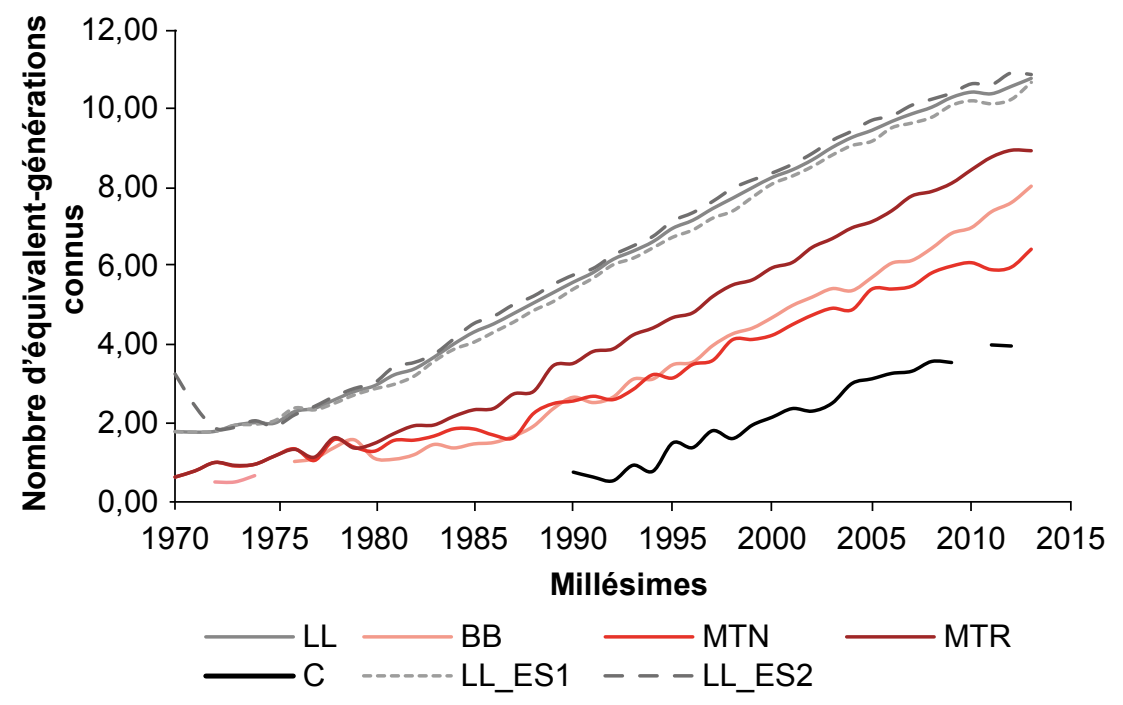

Figure 4. Proportions d'ancêtres connus en fonction du nombre de générations prises en compte pour les béliers du noyau de sélection, nés de 2000 à 2009, dans les cinq races ovines laitières françaises en sélection.

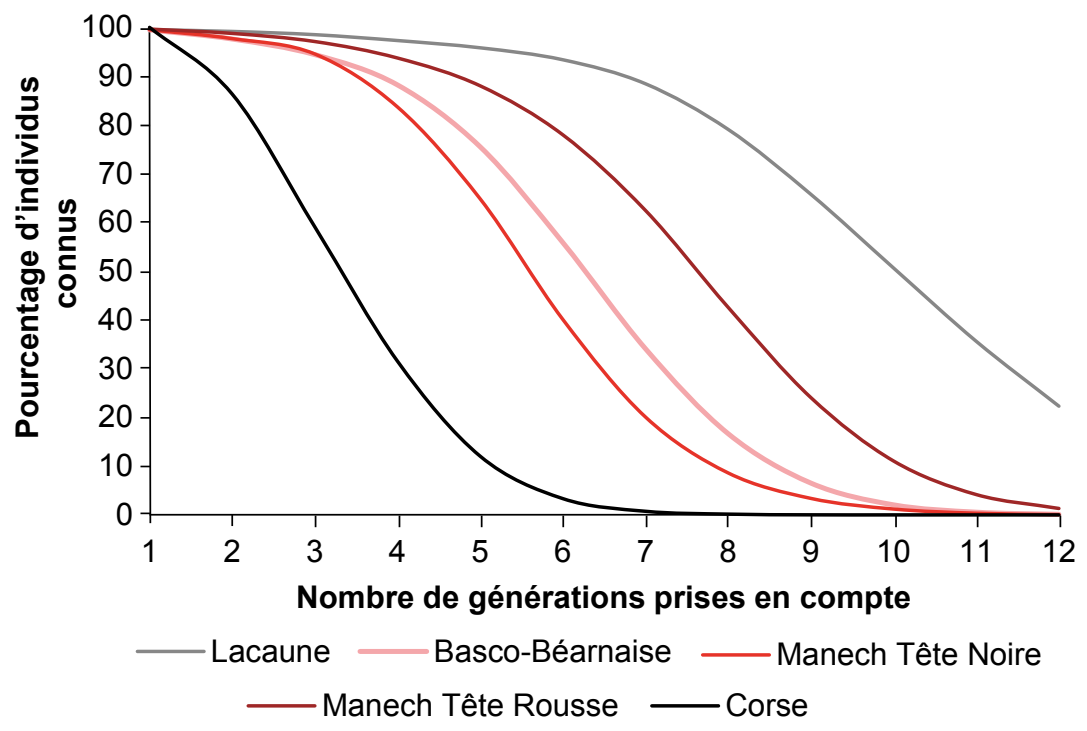


La profondeur des pedigrees de la race $L L$ est équivalente à celle des races bovines Prim'Holstein ou Normande, et des races caprines françaises Alpine et Saanen, tandis que Ngen en races ovines BB et MTR se rapproche de Ngen de la race bovine Montbéliarde (Idele, 2015). Compte tenu de la connaissance plus limitée des pedigrees en race Corse, I'analyse de variabilité génétique à partir de l'information généalogique est donc nettement moins informative, la consanguinité des béliers Corse étant de fait sous-estimée. Une étude à partir des données moléculaires provenant des 18500 génotypages disponibles (puce Illumina ovine SNP50), dont 488 mâles Corses nés entre 2001 et 2015, a donc été mise en place en ovin lait, et l'analyse est en cours.

\section{3. Étude de la consanguinité et des apparentements}

\subsection{Méthode}

La consanguinité (F) d'un individu est la probabilité qu'à un locus autosomal neutre vis-à-vis de la sélection, pris au hasard, cet individu ait reçu de ses parents deux gènes identiques qui leur a été transmis à chacun par un ancêtre commun. Dans une population fermée et en sélection, l'augmentation de consanguinité est inéluctable et est d'autant plus rapide que le nombre de reproducteurs est faible. Différentes méthodes de calcul ont été développées pour estimer ces coefficients de consanguinité, dont celle de Meuwissen et Luo (1992), et celle de Van Raden (1992). Cette dernière permet notamment de calculer la consanguinité à nombre de générations donné, ce qui permet de distinguer la consanguinité proche, qui résulte de l'accouplement entre proches parents ( 2 ou 3 dernières générations), de la consanguinité éloignée, imputable à des ancêtres plus éloignés et apparentés, et également de pouvoir comparer des races, pour une même période, à même profondeur de pedigrees fixée pour les calculs de consanguinité. Ces deux méthodes ont été utilisées ici à l'aide du package PEDIG (Boichard, 2002), logiciel à partir duquel ont été obtenus l'ensemble des indicateurs de variabilité génétique présentés dans cet article. L'étude de l'accroissement de la consanguinité par génération permet ensuite d'estimer l'effectif génétique $\mathrm{Ne}(\Delta \mathrm{F} /(1-\mathrm{F})=1 /(2 \mathrm{Ne}))$, (Boichard et al., 1996), qui correspond à la taille d'une population idéale présentant la même évolution asymptotique de consanguinité.

\section{2. Évolution de la consanguinité}

L'étude de la consanguinité moyenne par millésime montre une augmentation au cours du temps pour l'ensemble des races (figure 5). L'accroissement annuel de consanguinité en LL et MTR est relativement modéré et constant, respectivement $+0,08$ et $+0,11 \%$ de consanguinité par an entre 2000 et 2009 , soit $+0,29$ et $+0,42 \%$ par génération, ce qui correspond à des effectifs génétiques $(\mathrm{Ne})$ de 174 et 118 individus respectivement en LL et MTR (tableau 3). De tels effectifs génétiques (Ne) en races $\mathrm{LL}$ et MTR sont comparables respectivement à ceux estimés pour les races caprines Alpine (185) et Saanen (139), qui présentent par ailleurs des Ngen voisins dans cette même période (Idele, 2015).

Figure 5. Évolution de la consanguinité moyenne des béliers du noyau de sélection, nés de 1970 à 2013 dans les races Lacaune (LL), Basco-Béarnaise (BB), Manech Tête Noire (MTN), Manech Tête Rousse (MTR), Corse (C), et dans chacune des deux entreprises de sélection Lacaune (LL_ES1 et LL_ES2).

On tient compte ici de l'intégralité de l'information disponible dans les pedigrees.

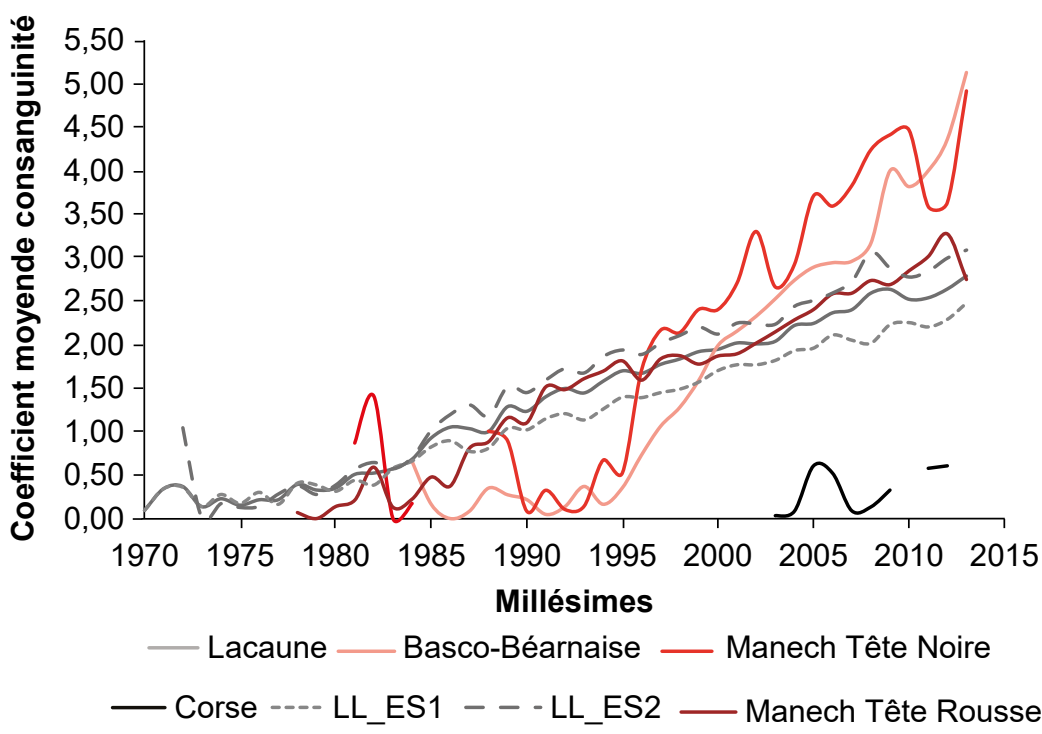


Tableau 3. Accroissement de consanguinité $(\Delta F)$ par an et par génération, effectif génétique estimé ( $\mathrm{Ne}$ ), et probabilité d'origine des gènes pour les béliers du noyau de sélection, nés entre 2000 et 2009 dans les races Lacaune (LL), Basco-Béarnaise (BB), Manech Tête Noire (MTN) et Manech Tête Rousse (MTR).

\begin{tabular}{|l|c|c|c|c|}
\hline & LL & BB & MTN & MTR \\
\hline$\Delta$ F/an & $+0,08 \%$ & $+0,18 \%$ & $+0,21 \%$ & $+0,11 \%$ \\
\hline Intervalle de génération & 3,60 & 4,15 & 4,84 & 3,97 \\
\hline$\Delta$ F/génération & $+0,29 \%$ & $+0,74 \%$ & $+1,02 \%$ & $+0,42 \%$ \\
\hline Effectif génétique (Ne) & 174 & 67 & 49 & 118 \\
\hline Fondateurs efficaces (Fe) & 202 & 69 & 42 & 122 \\
\hline Ancêtres efficaces (Ae) & 83 & 24 & 18 & 38 \\
\hline Rapport Ae/Fe & $41 \%$ & $35 \%$ & $43 \%$ & $31 \%$ \\
\hline $\begin{array}{l}\text { Contribution de l'ancêtre } \\
\text { le plus important }\end{array}$ & $4,9 \%$ & $12,9 \%$ & $16,5 \%$ & $10,8 \%$ \\
\hline $\begin{array}{l}\text { Nombre d'ancêtres } \\
\text { contribuant à 50 \% des gènes }\end{array}$ & 33 & 9 & 7 & 16 \\
\hline
\end{tabular}

ovins laitiers, nés entre 2000 et 2009 , a été calculée en bornant le nombre de générations prises en compte de 2 à 10 . Les consanguinités moyennes obtenues, notées vrd2 à vrd10, sont présentées en figure 6. Les valeurs nulles à quasi-nulles de vrd2 et vrd3 indiquent que toutes les ES respectent parfaitement la

règle des accouplements interdits avec l'absence de consanguinité proche. En revanche, alors que les consanguinités moyennes augmentent progressivement avec la prise en compte de la quatrième et la cinquième génération (vrd4 et vrd5 $\leq 1 \%$ ) en LL et MTR, un accroissement plus important de la

Figure 6. Comparaison de l'évolution de la consanguinité des béliers du noyau de sélection, nés de 2000 à 2009, dans quatre races ovines laitières, selon le nombre de générations prises en compte.

Ngen correspond au nombre d'équivalent de générations connues, Fà la consanguinité moyenne de la population étudiée en utilisant toute l'information généalogique disponible, et vrd2-vrd10 à la consanguinité moyenne en ne tenant compte que des 2-10 dernières générations (vrd2 nulle ou quasi nulle à chaque fois).

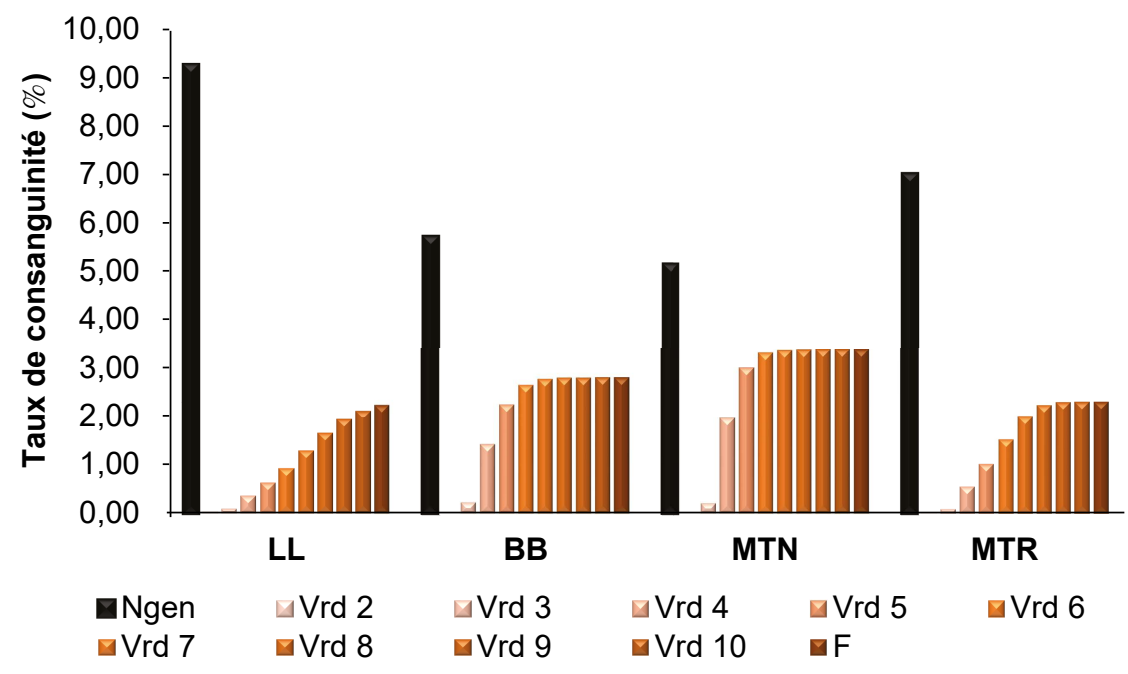

consanguinité est observé en BB et MTN dès la prise en compte de la quatrième génération ( $\mathrm{vrd} 4=1,4$ et $2 \%$ respectivement) puis de la cinquième génération (vrd5 $=2,2 \%$ et $3 \%$ respectivement). Ce constat montre que la gestion actuelle des accouplements interdits ne suffit plus à pallier l'augmentation de consanguinité dans ces deux races, du fait du niveau d'apparentement plus marqué à partir des arrière-grands-parents qui résulte d'un nombre insuffisant de familles de PAB dans les races BB et MTN. En effet, dans ces deux races, la petite taille du noyau de sélection et la concentration de la campagne d'IA sur quelques semaines rendent moins facile la gestion des PAB, d'où l'apparition de déséquilibres entre familles élites, d'autant que la maîtrise de I'IA est plus problématique dans ces deux populations.

\subsection{Apparentements des mâles élites}

Les mâles élites, ou PAB, sont à l'origine de l'ensemble du haras de béliers d'IA, et de fait, d'une forte proportion des femelles du CLO. L'étude des apparentements entre PAB permet donc d'avoir une bonne idée de l'évolution à venir de la variabilité génétique dans la population considérée. Les résultats obtenus sont présentés dans le tableau 4. Quelle que soit la race/ES étudiée, les apparentements moyens entre $P A B$ tendent à augmenter progressivement au cours du temps, pour atteindre, dans la dernière décennie, 2,84 à 3,43 \% selon l'ES LL, versus $5,64,5,75$ et $3,50 \%$ respectivement en BB, MTN et MTR. II est en outre intéressant de noter que l'apparentement entre les deux populations LL est nettement plus faible qu'intra ES LL et qu'il tend à diminuer au cours du temps pour se limiter à $0,50 \%$ à la dernière décennie, reflet du peu d'échanges de gènes entre les deux ES depuis maintenant plus de quatre décennies. La MTR présente des apparentements moyens entre PAB semblables à ceux observés dans la deuxième ES Lacaune $(3,50 \%)$, avec toutefois une profondeur de pedigrees un peu moindre : il est donc nécessaire de rester vigilant en MTR pour ne pas risquer de basculer vers la situation des BB et MTN, présentant des parentés moyennes entre $\mathrm{PAB}$ contemporains respectivement de 5,64 et $5,75 \%$, avec des 
Tableau 4. Évolution (en \%) de la parenté moyenne entre les Pères à Béliers (PAB), au cours des trois dernières décennies. Ces parentés sont calculées intra race en Basco-Béarnaise (BB), Manech Tête Noire (MTN), et Manech Tête Rousse (MTR), et intra Entreprise de Sélection (ES) pour la race Lacaune (LL). L'apparentement moyen entre les PAB des deux ES Lacaune est également donné (LL_INTER ES).

\begin{tabular}{|l|c|c|c|}
\hline & $\mathbf{1 9 8 0 - 1 9 8 9}$ & $\mathbf{1 9 9 0 - 1 9 9 9}$ & $\mathbf{2 0 0 0 - 2 0 0 9}$ \\
\hline LL_ES1 & 1,70 & 2,18 & 2,84 \\
\hline LL_ES2 & 2,01 & 2,72 & 3,43 \\
\hline LL_inter ES & 0,70 & 0,50 & 0,50 \\
\hline BB & 2,05 & 4,07 & 5,64 \\
\hline MTN & 2,77 & 6,28 & 5,75 \\
\hline MTR & 2,23 & 3,17 & 3,50 \\
\hline
\end{tabular}

profondeurs de pedigrees comparables à celle de la MTR et inférieures à celle de la LL (figures 3 et 4 ).

Au vu de ces résultats, un focus a été réalisé sur le cheptel de $\mathrm{PAB}$ des races $\mathrm{BB}$ et MTN. Compte tenu de la plus petite taille de leurs noyaux de sélection, le nombre de $P A B$ utilisés chaque année est plus limité (une vingtaine en $B B$ et une quinzaine en MTN) qu'en races LL ou MTR (entre 30 et 35). De plus, la saisonnalité de la campagne d'IA est plus marquée dans ces deux races, de sorte qu'avec des inséminations effectuées avec de la semence fraîche, cela tend à favoriser les $\mathrm{PAB}$ qui produisent le plus de semence durant cette courte période $d^{\prime} \mid A$, et qui procréent ainsi plus de fils. Le respect d'un usage équilibré des $P A B$, règle déterminante, n'est donc pas toujours respectée. Au fil des générations, il apparaît donc un déséquilibre de plus en plus marqué entre les familles de $\mathrm{PAB}$, à tel point qu'en $\mathrm{BB}$ aujourd'hui, la moitié des mâles élites est issue d'une seule et même famille.

\section{Probabilité d'origine des gènes}

\subsection{Méthode}

La probabilité d'origine des gènes est issue de l'analyse de la provenance des gènes d'un groupe d'individus en considérant qu'un gène autosomal neutre quelconque est transmis par son père ou sa mère avec une probabilité de 0,5 , par un de ses grands-parents avec une probabilité de 0,25. En remontant ainsi dans le pedigree de chaque individu, il est alors possible d'estimer la contribution à son génome de chacun de ses ancêtres connus (James, 1972 et Vu Tien Khang, 1983). Sont considérés comme fondateurs l'ensemble des ancêtres dont les deux parents sont inconnus, et qui sont à l'origine de tous les gènes actuels. Or, les contributions de ces fondateurs ne sont pas équilibrées, d'où l'estimation du nombre de fondateurs efficaces Fe (Lacy, 1989), corress'ils avaient tous la même contribution, conduiraient à la même variabilité génétique dans la population considérée. La contribution marginale de chaque ancêtre au pool de gènes actuels peut également être estimée, et comme précédemment, on peut définir le nombre d'ancêtres efficaces Ae (fondateurs ou non). L'estimation des nombres de fondateurs et d'ancêtres efficaces est dépendante de la connaissance des généalogies. Cependant, l'étude du rapport Ae/Fe permet de mettre en évidence la présence ou non de goulots d'étranglement, le rapport classique dans une population de ruminants sélectionnée se situant autour de 40 à $50 \%$.

\subsection{Fondateurs efficaces et ancêtres efficaces}

Le nombre de fondateurs dans une population est systématiquement pondant au nombre de fondateurs qui, surestimé en raison de la connaissance partielle des généalogies et ce nombre cache une grande disparité de contributions ; il est donc plus intéressant de considérer le nombre de fondateurs efficaces et/ou d'ancêtres efficaces (tableau 3). Pour les béliers de chaque noyau de sélection, nés de 2000 à 2009 , le nombre de fondateurs efficaces varie de 42 en MTN à 202 en LL, alors que le nombre d'ancêtres efficaces, plus petit, varie de 18 en MTN à 83 en LL (en accord avec les résultats de Palhière et al. (2000)). Le rapport Ae/Fe, qui varie de 31 à $43 \%$ selon les races, témoigne de l'existence de goulots d'étranglement. Ces derniers sont mis en évidence par l'étude des contributions marginales: l'ancêtre le plus important contribue à environ $5 \%$ des gènes en $L L$, alors qu'il contribue de 10 à $16,5 \%$ des gènes en ROLP (tableau 3). Par ailleurs, le nombre d'ancêtres qui contribuent à $50 \%$ des gènes varie de 7 à 33 selon les races. Les présents résultats des béliers nés de 2000 à 2009 étant proches de ceux obtenus par Palhière et al. (2000) pour les béliers nés de 1989 à 1999, il est donc possible de conclure que le plan de sélection pour la résistance à la tremblante mis en place en ovins laitiers à la fin de la décennie 2000 a été bien géré du point de vue de la variabilité génétique et n'a pas induit de goulot d'étranglement. Ces résultats viennent corroborer les résultats obtenus par Brochard et al. (2006).

En outre, le nombre de fondateurs efficaces et d'ancêtres efficaces, respectivement 202 et 83 en race $L L, 122$ et 38 en race MTR, est au moins aussi satisfaisant qu'en races laitières caprines, respectivement 173 et 38 en race Alpine et 78 et 37 en race Saanen, et meilleur qu'en races bovines laitières, respectivement 83 et 20 en race Prim'Holstein et 80 et 21 en race Normande (Idele, 2015).

\section{Appui à la gestion de la variabilité génétique auprès des schémas de sélection}

Suite à l'analyse rétrospective de la variabilité génétique qui vient d'être présentée, différents appuis aux maîtres 
d'œuvre des schémas ont été réalisés au cours des dernières années, soit ponctuellement (cas de la BB) soit en routine pour l'aide au choix des béliers aux étapes clé du schéma de sélection.

\subsection{Travail réalisé en race Basco-Béarnaise}

Une attention particulière a été portée aux ROLP, et notamment à la $B B$, en raison de l'accroissement important de consanguinité observé par générations. Cet accroissement résulte de la priorité supérieure qui a été accordée au gain génétique annuel (3 litres de lait environ), par rapport à la recherche d'un usage aussi équilibré que possible des familles élites de $P A B$, règle clé de la gestion de la variabilité génétique en situation d'IA en semence fraîche. En effet, actuellement la vingtaine de $\mathrm{PAB}$, en rapport avec la taille du noyau de sélection du schéma BB (tableau 1), n'est répartie qu'en 4 familles élites, versus au moins 8 à 10 si l'usage avait été équilibré : il y a donc actuellement, en race $B B, 2$ à $10 P A B$ par famille, alors que pour une ES LL, les 30 à $40 P A B$ sont répartis en 20 à 30 familles de $P A B$, soit 1 à 2 PAB par famille.

En 2014, une analyse des PAB a donc été menée avec le CDEO d'Ordiarp (Centre Départemental de l'Élevage Ovin), maître d'œuvre du schéma de sélection $\mathrm{BB}$. Elle confirme l'existence de seulement 4 familles de $P A B$, très déséquilibrées, et $11 \mathrm{PAB}$ sur les 21 appartiennent à une même famille et présentent une parenté moyenne entre eux de $11,7 \%$, donc de type demi-frères ou oncle-neveu. Deux autres familles comptent chacune 4 PAB et les apparentements intra-famille sont respectivement de 16,0 et $13,6 \%$. II existe également des liens entre ces 4 familles, de sorte que l'apparentement moyen entre tous les PAB de 2014 est égal à 7,6 $\pm 4,6 \%$, et la consanguinité moyenne à $4,1 \pm 1,5 \%$. Ces constats aboutissent à un nombre croissant d'accouplements interdits dans cette race, ce qui pose des problèmes pour programmer les accouplements raisonnés.

Une réflexion a donc été menée pour proposer une nouvelle liste de PAB afin de réaliser un compromis mieux équilibré entre gain génétique et gestion de la variabilité génétique. Les mâles les plus apparentés aux autres et les plus consanguins (coefficient de parenté $\geq 8 \%$ ou coefficient de consanguinité $\geq 5 \%$ ) ont été écartés du pool de PAB et ont été remplacés par des béliers améliorateurs (Pères à Filles ou PAF).

L'index de synthèse (ISOL) moyen des nouveaux PAB ainsi choisis est inférieur de 28 points par rapport à celui du choix initial, et les changements effectués permettent de réduire l'apparentement moyen entre $\mathrm{PAB}$ à $6,1 \pm 3,4 \%$, et de faire baisser la consanguinité moyenne des PAB à $3,6 \pm 1,0 \%$. Ainsi, en consentant à une baisse moyenne d'index de $8 \%$, l'apparentement moyen entre PAB est réduit de $19 \%$, et leur consanguinité moyenne de $12 \%$. Par ailleurs, l'apparentement des PAB intra famille est réduit par rapport au choix initial et l'apparentement entre familles prises 2 à 2 est globalement réduit.

Dans cette configuration, une cinquième famille a été ajoutée. La famille majeure étant actuellement impossible à contourner, compte tenu du nombre de ses représentants et de leur niveau génétique, le choix a été fait de la redécouper en deux groupes d'apparentés distincts, les plus éloignés possibles entre eux, afin de les faire évoluer en deux familles distinctes au fil du temps. Le nombre de familles passe donc de 4 à 6 , avec 2 à 4 PAB par familles. II s'agit donc d'une nouvelle orientation significative en faveur de la gestion de la variabilité génétique, en gardant en point de mire l'objectif de tendre vers 8 à 10 familles de $P A B$, avec 1 à 2 béliers d'IA élites par famille.

Ces propositions ont été soumises aux gestionnaires du schéma ainsi qu'aux principaux éleveurs sélectionneurs avant d'être validées puis mises en application. Il convient de maintenir de telles évolutions du choix des PAB et leur éclatement en un plus grand nombre de familles mieux équilibrées, pendant au moins une décennie, pour améliorer de façon durable la situation de la variabilité génétique en $B B$.

\subsection{Indicateurs fournis aux gestionnaires des schémas}

Compte tenu des résultats obtenus lors de ce bilan rétrospectif et des observations faites lors du travail pour le choix des PAB BB et MTN, des indicateurs de variabilité génétique sont fournis en routine aux gestionnaires du schéma pour les aider à faire leur choix aux différentes étapes de la sélection. Tout d'abord, les taux de consanguinités de tous les mâles d'IA et leur répartition par famille sont fournis, ainsi que la consanguinité moyenne des PAB, leur parenté moyenne et leurs parentés deux à deux. Si les résultats semblent trop élevés ou si les familles sont trop déséquilibrées, une discussion est alors menée avec le gestionnaire du schéma. Afin d'aider à la gestion des accouplements raisonnés et aux choix des agneaux nés de ces accouplements, la liste des apparentements entre chaque $P A B$ et chaque mère élite est fournie aux gestionnaires. Enfin, lors du choix des agneaux pour I'IA, l'équilibre entre les familles, la consanguinité moyenne et l'apparentement moyen entre les candidats proposés, puis retenus sont également vérifiés.

\subsection{Gestion \\ de la variabilité avec l'arrivée de la Sélection Génomique}

Alors que les indicateurs de variabilité génétique poussent à être vigilant sur la gestion des reproducteurs dans les schémas de sélection classique et à mettre en place un suivi plus poussé dans les petites races, qu'en sera-t-il dans un schéma de sélection génomique?

En effet, la sélection génomique a été mise en place en ovin lait dès 2015 en race Lacaune, et en 2017 en ROLP. Dans ces schémas génomiques, les IA de « testage » sont remplacées par des $\mathrm{I} A$ « d'entretien de la population de référence ", réalisées exclusivement en CLO, permettant de disposer des phénotypes nécessaires pour actualiser les équations de prédiction génomique (Astruc et al., 2016). Les béliers sont ensuite diffusés comme PAB ou PAF génomiques, sans attendre les résultats des performances 
de leurs filles, ce qui permet de réduire le nombre de béliers en centre d'IA. Cependant, la suppression de la période d'attente des résultats de testage ne permettant pas de compenser complètement les coûts générés par le génotypage d'un nombre important d'agneaux (Buisson et al., 2014), un effort particulier a été porté en vue de la maximisation de la production de semence, dans le but de réduire le plus possible le nombre de béliers en centre $d^{\prime} \mid A$.

En LL, le nombre de mâles d'IA sélectionnés par génération a ainsi été divisé par deux, réduisant de moitié la proportion d'IA en CLO réalisée par les agneaux et complément éventuel en antenais : de 50 à $25 \%$. Dans le schéma classique, les IA CLO étant réalisées uniquement, d'une part par des agneaux en testage et éventuellement par des antenais en complément de testage à hauteur de $50 \%$, et d'autre part par les $P A B$, également à hauteur de $50 \%$. La question de qualifier ou non plus de $P A B$ s'est donc posée, pour assurer les $75 \%$ d'IA restantes en situation génomique (au lieu de $50 \%$ dans le schéma classique). Compte tenu de la réduction par deux du nombre de mâles d'IA par cohorte (fils de $P A B$ ), augmenter le nombre de $P A B$ reviendrait à retenir très peu de fils par $\mathrm{PAB}$, au point d'accroître le risque de disparition de certaines familles de PAB par rapport à la situation du schéma classique. En optant pour le maintien du nombre de $P A B$, les gestionnaires des schémas LL ont choisi de préserver la recherche de la gestion équilibrée des familles de $P A B$ pour assurer leur pérennité. En conséquence, les meilleurs PAF améliorateurs sont alors utilisés en CLO pour assurer les $25 \%$ d'IA restantes, sans augmentation donc du nombre de PAB par rapport au schéma classique.

En ROLP, compte tenu du complément de testage plus systématique à 18 mois et d'une production de semence par bélier plus faible, la réduction du nombre d'agneaux par génération est moindre (baisse de $20 \%$ en moyenne). Là encore, les gestionnaires des schémas ont fait le choix, pour l'instant, de maintenir le nombre actuel de PAB et donc d'utiliser les meilleurs PAF améliorateurs en CLO pour assurer les compléments $d^{\prime} \mid A$ en situation génomique, l'augmentation du nombre de PAB n'étant pas acceptable puisqu'elle impliquerait d'abaisser le seuil d'index actuel, et donc le niveau génétique moyen des $P A B$, et de diminuer le nombre de fils par PAB candidats à devenir béliers d'IA, donc à augmenter le risque de déséquilibre entre familles de $P A B$, qui est déjà un problème en schéma classique en ROLP. Un tel choix est pleinement justifié en race MTR, dont le compromis entre gain génétique annuel et préservation de la variabilité génétique est assez proche de celui d'une ES LL. En revanche, le statut quo sur le maintien du nombre de PAB en BB et MTN ne se justifie que si le processus amorcé en 2014, consistant à ré-éclater le cheptel actuel de PAB en un plus grand nombre de familles mieux équilibrées, réussit dans la décennie en cours. Le cas échéant, en races BB et MTN, un meilleur compromis entre gain génétique et variabilité génétique pourrait passer par une augmentation du nombre total de PAB en incorporant des PAF dans le pool des $P A B$ (jusqu'à $75 \%$ d'IA en $P A B$ ), ce qui pourrait favoriser l'augmentation du nombre de familles de PAB et un meilleur équilibre entre elles. Cela correspondrait alors à une orientation plus favorable au maintien de la variabilité génétique à long terme qu'au seul progrès génétique à court terme.

On peut remarquer enfin que la règle essentielle d'usage équilibré des PAB à toutes les étapes du schéma de sélection s'impose au plan opérationnel en raison de la pratique exclusive de I'IA en semence fraîche en ovins laitiers. En effet, le recours à la méthode performante de Sélection à Parenté Minimum (SPM), pour la gestion combinée du progrès génétique et de la variabilité génétique, proposée par Colleau et al. (2004), suppose une contribution très déséquilibrée intra-année de l'usage des mâles élites (pères à fils), qui ne peut se planifier et se pratiquer qu'en situation d'IA en semence congelée : cette méthode, pratiquée avec succès en races caprines laitières Alpine et Saanen depuis 2016, a été décrite par Palhière et al. (2014).

Par ailleurs, le génotypage des cohortes de béliers permet de disposer d'une information supplémentaire pour étudier la variabilité génétique à partir de données moléculaires (puce
Illumina ovine SNP50). Une analyse est actuellement en cours pour étudier la consanguinité et la parenté génomique moyenne des individus et ainsi améliorer la gestion de la variabilité génétique en s'affranchissant des pedigrees.

\section{Conclusion}

La préservation d'une variabilité génétique satisfaisante est un enjeu majeur pour les populations en sélection, aussi bien pour garder une bonne capacité d'adaptation à de nouveaux systèmes de production pour les caractères sélectionnés, que pour permettre une réorientation des objectifs de sélection en faveur de nouveaux caractères. Compte tenu de la diminution de l'intervalle de génération père-fils et de l'augmentation du progrès génétique prévu dans le cadre d'un schéma génomique, il est d'autant plus important de connaître et de gérer au mieux la variabilité génétique au sein de chaque race locale de brebis laitières françaises en sélection.

La présente étude a ainsi permis de vérifier que la variabilité génétique a été bien gérée en race LL grâce à un usage équilibré des familles de $P A B$, facilitée par l'importance des effectifs de mâles gérés. En outre, les échanges de semence et de reproducteurs étant très limités entre les deux ES LL depuis plus de 40 ans, chacune d'elle est potentiellement un réservoir important de variabilité génétique pour l'autre, ce qui constitue un atout indéniable pour la sécurité et la gestion de la diversité génétique à long terme de cette race. La variabilité génétique est également assez bien gérée en MTR, avec un noyau de sélection de taille comparable à celui d'une ES LL. Toutefois, une amélioration de la maîtrise de l'équilibre entre familles de PAB est possible, à l'instar de la situation Lacaune où chaque famille élite d'IA ne comprend qu'un ou deux PAB. L'accroissement de consanguinité est effectivement bien modéré et bien maîtrisé en MTR, mais les résultats de l'étude de probabilité d'origine des gènes invitent à rester vigilant dans cette race, afin de ne pas basculer dans une situation défavorable. En revanche, les règles de gestion actuelle de la variabilité génétique, et surtout leur mise en œuvre, semblent critiques dans les 
petites populations (BB et MTN) : la taille plus limitée de leur noyau de sélection, la très forte concentration de leur saison d'IA, la moins bonne réussite à I'IA, et le travail en semence fraîche sont autant de facteurs qui ont contribué à un accroissement plus rapide de l'apparentement et donc de la consanguinité. Cette situation résulte de déséquilibres importants entre les familles de PAB, qui se traduisent en BB et MTN par un apparentement trop important entre tous les $P A B$, appartenant à un nombre trop limité de familles de PAB, au moins deux fois moins que le nombre attendu de familles à usage plus équilibré entre elles, à toutes les étapes du schéma de sélection.

L'accompagnement mis en place auprès des ES devrait permettre de les aider à mieux gérer la variabilité génétique via un choix plus éclairé des mâles reproducteurs et des accouplements, et une gestion de l'équilibre entre familles plus stricte dans le cadre du schéma génomique. Si le choix de maintien de l'effectif de PAB est arrêté pour toutes les races en sélection génomique, il pourrait toutefois être revu en BB et MTN, si le travail en cours, visant l'augmentation du nombre de familles élites et un meilleur équilibre entre elles, n'est pas assez efficace dans les prochaines années.

Le déploiement de la sélection génomique pose néanmoins la question de la prise en compte des données moléculaires pour étudier la variabilité génétique. En effet, outre la possibilité de révéler l'originalité des individus en s'affranchissant des généalogies, il a été démontré que pour contrôler de manière efficace la consanguinité d'une population en sélection génomique, il est nécessaire de se baser sur les informations moléculaires, I'utilisation des pedigrees impliquant une surestimation de l'accroissement de consanguinité (Sonesson et al., 2012). Le bilan de la variabilité génétique des races ovines laitières françaises en sélection, fondé pour l'instant sur des indicateurs issus de généalogies, sera complété par des indicateurs issus de génotypages dans le cadre d'un travail en cours. Un bilan à venir de l'évolution au fil des dernières décennies de la variance génétique additive des caractères en sélection, estimée à partir d'informations phénotypiques et généalogiques, contribuera également à établir un état des lieux complet de la variabilité génétique des ovins laitiers français.

\section{Références}

Astruc J.M., Lagriffoul G., Larroque H., Legarra A., Moreno C., Rupp R., Barillet F., 2010. Use of genomic data in French dairy shep breeding programs: results and prospects. Proc. $37^{\mathrm{e}}$ session d'ICAR, Riga, Lettonie. ICAR, Rome, Italie.

Astruc J.M., Baloche G., Buisson D., Labatut J., Lagriffoul G., Larroque H., Robert-Granié C., Legarra A., Barillet F., 2016. La sélection génomique des ovins laitiers en France. In: Brebis laitières en France: 50 ans de recherche et développement. Barillet F., Hassoun P., Astruc J.M., Lagriffoul G., Morin E. (Éds). Dossier, INRA Prod. Anim., 29, 41-56.

Barillet F., Vu Tien Khang J., Roussely M., Poivey J.P., Chevalet C., Elsen J.M., de Rochambeau H., 1989. Variabilité génétique dans les noyaux de sélection des ovins laitiers de race Lacaune: une étude rétrospective. In : Bureau des Ressources Génétiques (Éds), Actes du Colloque «La gestion des ressources génétiques des espèces animales domestiques », Paris, France, 72-80.

Barillet F., Palhière I., Astruc J.M., Brochard M., Baelden M., Aguerre X., Fidelle F., Arranz J.M., Belloc J.P., Briois M., Fregeat G., Soulas C., Andreoletti 0., Corbière $F$., Schelcher F., 2004. Le programme français d'éradication de la tremblante du cheptel ovin fondé sur I'utilisation de la génétique. In : Numéro hors-série, Encéphalopathies spongiformes transmissibles animales. Ducrot C., Charley-Poulain J., Aynaud J.M. (Éds). INRA Prod. Anim., 17, 87-100.

Boichard D., 2002. PEDIG: a Fortran package for pedigree analysis suited for large populations. In: Proc. 7th World Congr. Genet. Appl. Livest. Prod., Montpellier, France.

Boichard D., Maignel L., Verrier E., 1996. Analyse généalogique des races bovines laitières françaises. INRA Prod. Anim., 9, 323-335.

Brochard M., Palhièrel., VerrierE., Moazami-Goudarzi K., Neuts E., Amigues Y., Barillet F., Bed'hom B., Bibe B.,
Bouix J., Cribiu E.P., François D., Leymarie C., Tantano T., 2006. Impact d'une sélection forte pour un gène majeur sur la variabilité et les niveaux génétiques : cas de la sélection pour la résistance à la tremblante ovine. Renc. Rech. Rum., 13, 231-234.

Buisson D., Lagriffoul G., Baloche G., Aguerre X., Boulenc P., Fidelle F., Frégeat G., Giral-Viala B., Guibert P., Panis P., Soulas C., Astruc J.M., Barillet F., 2014. Genomic breeding schemes in French Lacaune and Manech dairy sheep: design and expected genetic gain. In: Proc. World Congr. Genet. Appl. Livest. Prod., Vancouver, Canada, 898.

Colleau J.J., Moureaux S., Briend M., Bechu J., 2004. A method for the dynamic management of genetic variability in dairy cattle. Genet., Sel., Evol., 36, 373-394.

de Rochambeau H., Verrier E., Bidanel J.P., Maignel L., Labroue F., Tribout T., Palhière I., Astruc J.M., Barillet F., Chapuis H., 2003. Mise en place de procédures de suivi de la variabilité génétique des populations domestiques sélectionnées et établissement de guides de gestion : application aux ovins laitiers et aux porcs. In : les Actes du BRG, 4, 17-34.

Idele, 2015. Observatoire Varume. http://idele.fr/ filieres/caprin/publication/ldeleSolr/recommends/ varume-resultats-2015.html

Idele et CNBL, 2015. Groupe Génétique du CNBL : Journées des 15 et 16 octobre 2014. Collection Résultats. Compte-rendu n 0015202 001, janvier 2015.

Idele et CNBL, 2017. Groupe Génétique du CNBL : Journées des 18 et 19 octobre 2016. Collection Résultats. Compte-rendu n 0017202 003, janvier 2017.

James J., 1972. Computation of genetic contributions from pedigrees. Theor. Appl. Genet., 42, 272-273.
Lacy R.C., 1989. Analysis of founders representation in pedigrees: founder equivalents and founder genome equivalents. Z00. Biol., 8, 111-123.

Meuwissen T.H.E., Luo Z., 1992. Computing inbreeding coefficients in large populations. Genet. Sel. Evol., 24, 305-313.

Palhière I., Barillet F., Astruc J.M., Aguerre X., Belloc J.P., Briois M., Fregeat G., Bibe B., Rochambeau H., Boichard D., 2000. Analyse de la variabilité génétique des races ovines laitières Basco-Béarnaises et Manech à partir des informations généalogiques. Renc. Rech. Rum., 7, 153-156.

Palhière I., Clément V., Martin P., Colleau J.J., 2014. Bilan de la méthode de Sélection à Parenté Minimum après 6 ans d'application dans le schéma de sélection caprin. Renc. Rech. Rum., 21, 253-256.

Sonesson A.K., Woolliams J.A., Meuwissen T.H.E., 2012. Genomic selection requires genomic control of inbreeding. Genet. Select. Evol., 44, 27.

Sørensen A.C., Sørensen M.K., Berg P., 2005. Inbreeding in Danish dairy cattle breeds. J. Dairy Sci., 88, 1865-1872.

Stachowicz K., Sargolzaei M., Miglior F., Schenkel F.S., 2011. Rates of inbreeding and genetic diversity in Canadian Holstein and Jersey cattle. J. Dairy Sci., 94, 5160-5175.

Van Raden P.M., 1992. Accounting for inbreeding and crossbreeding in genetic evaluation for large populations. J. Dairy Sci., 75, 3136-3144.

Vu Tien Khang J., 1983. Méthodes d'analyse des données démographiques et généalogiques dans la population d'animaux domestiques. Genet. Sel. Évol., 15, 263-298. 


\section{Résumé}

La variabilité génétique est la marge de progression, le carburant de la sélection, et il apparaît d'autant plus important d'en réaliser un état des lieux que l'arrivée de la sélection génomique chez les ovins laitiers en France devrait s'accompagner d'une diminution de l'intervalle de génération et d'une augmentation du progrès génétique. Une analyse rétrospective de la gestion de la variabilité génétique des races ovines laitières françaises en sélection à partir des informations généalogiques a été réalisée sur les 40 dernières années. La variabilité génétique est bien maîtrisée dans les races Lacaune (LL) et Manech Tête Rousse (MTR) : accroissement de consanguinité respectivement de + 0,29 et + 0,42\% par génération entre 2000 et 2009, avec toutefois la possibilité d'améliorer la gestion de l'équilibre entre familles élites en MTR. En revanche, la situation apparaît moins favorable en races Basco-Béarnaise (BB) et Manech Tête Noire (MTN) : accroissement de consanguinité respectivement de $+0,74$ et $+1,02 \%$ par génération entre 2000 et 2009. Cette situation résulte du nombre au moins deux fois trop faible de familles de Pères à Béliers (PAB) par rapport au nombre permis par la taille des noyaux de sélection BB et MTN. Elle est imputable à un trop fort déséquilibre dans l'usage des familles de PAB, qui découle pour partie de la durée très courte de la période d'Insémination Artificielle (IA) (semence fraîche), et de la moindre fertilité à l'IA. Des travaux ont été menés avec les gestionnaires de ces schémas pour augmenter dès 2015 le nombre de familles de $P A B$, et fournir, à chaque étape de sélection, des indicateurs de variabilité génétique pour gérer de façon plus équilibrée ces familles de PAB. La profondeur des pedigrees de la race Corse est insuffisante pour permettre une analyse pertinente des indicateurs issus des généalogies. Des travaux sont en cours dans les cinq races afin d'étudier la variabilité génétique à partir des données SNP («Single Nucleotid Polymorphism ») des quelques 18500 génotypages disponibles pour la sélection génomique des races LL, BB, MTR et MTN, ou réalisés spécifiquement pour cet objectif en race Corse.

\section{Abstract}

\section{Overview and perspectives of the management of genetic diversity in French dairy sheep}

Management of genetic diversity is a major concern in animal breeding, especially as genomic selection starting in French dairy sheep will reduce generation interval and increase genetic progress. Hence, it appeared important to assess the evolution of genetic diversity over the last decades using pedigree information to better monitor the breeding schemes. The results obtained in the Lacaune (LL) and Ref-Faced Manech breed (MTR) are satisfying: respectively +0.29 and $+0.42 \%$ of inbreeding increase per generation over 2000-2009, even though it is still possible to better balance elite families in MTR. On the contrary, results from Basco-Bearnaise (BB) and Black-Faced Manech breeds (MTN) underline a less efficient management of genetic diversity: respectively +0.74 and $+1.02 \%$ of inbreeding increase per generation over 2000-2009. This situation results from the too small number of sires of ram families $(P A B)$ compared to the number allowed by the size of the nucleus of selection BB and MTN. This is a consequence of an imbalanced use of PAB families due to the very short duration of the Al period (fresh semen) and the lower Al fertility in these two breeds. Studies were carried out with the managers of breeding societies. This was done in order to increase the number of PAB families and to provide at each step of selection, the indicators of genetic variability allowing a more balanced management of these PAB families. Corse breed pedigrees are not extensive enough to allow a relevant pedigree analysis. Studies are on-going in these five breeds to assess genetic variability based on SNP (Single Nucleotid Polymorphism) from the 18,500 genotypes available with genomic selection.

BUISSON D., ASTRUC J.-M., BARILLET F., 2018. Bilan et perspectives de la gestion de la variabilité génétique des ovins laitiers en France. INRA Prod. Anim., 31, 1-12. 